\title{
Griscelli syndrome subtype 2 with hemophagocytic lympho- histiocytosis: A case report and review of literature
}

\author{
Priyanka Minocha, Richa Choudhary*, Anika Agrawal, Sadasivan Sitaraman \\ Department of Pediatrics, Sawai Man Singh Medical College and Hospital, Jaipur, Rajasthan, India.
}

\begin{abstract}
Summary Griscelli syndrome (GS) is a rare autosomal recessive disorder resulting in pigmentary dilution of the skin and hair with variable phenotypes depending upon subtypes. Mutations in 3 distinct genes $M Y O 5 A, R A B 27 A, M L P H$ are responsible for 3 subtypes (GS1, GS2, and GS3) of GS respectively. GS subtype 2 commonly develops hemophagocytic lymphohistiocytosis (HLH) and recurrent infections due to immunodeficiency. We hereby report a 20 month old male child presenting with silvery gray hair, hypomelanosis and features of hemophagocytosis. The diagnosis of a type 2 GS was made in response to a set of clinical features: hypopigmentation of skin and the silvered reflection of the hair, absence of psychomotor retardation, the occurrence of an accelerated phase (hemophagocytosis) and, above all, a pathognomonic appearance by microscopic examination of a hair. The absence of giant granules in the nucleated cells made it possible to eliminate Chediak-Higashi syndrome, which shares a close clinical spectrum with GS. This case promotes awareness about this rare case of GS as a high indicator of suspicion about this potentially fatal condition and aids in prompt diagnosis and foresees complications. Early bone marrow transplant is the only curative treatment for GS-2.
\end{abstract}

Keywords: Griscelli syndrome, silvery grey hairs, hemophagocytic lymphohistiocytosis, chediakhigashi syndrome, immunodeficiency

\section{Introduction}

Griscelli syndrome (GS) is a rare autosomal recessive disorder resulting in pigmentary dilution of the skin and hair with variable phenotypes dependent upon subtypes. It is also known as hypopigmentation immunodeficiency disease and partial albinism with immunodeficiency. The most prominent features are silvery gray hair and hypopigmentation of skin. Mutations in three different genes- $M Y O 5 A, R A B 27 A$, and $M L P H$ are responsible for the 3 subtypes of GS respectively. GS subtype 1 usually manifests with primary dysfunction of the central nervous system without immunological involvement. Subtype 2 is characterized by severe immunological

Released online in J-STAGE as advance publication January 24, 2017.

*Address correspondence to:

Dr. Richa Choudhary, Department of Pediatrics, SPMCHI, Sawai Man Singh Medical College and Hospital, Jaipur, Rajasthan 302004, India.

E-mail: richa_8@yahoo.co.in impairment and commonly develops hemophagocytic lymphohistiocytosis (HLH) and recurrent infections while subtype 3 manifests with only partial albinism. Depending on the subtype, the prognosis of GS is variable. There is no specific treatment for GS-1 and prognosis depends on the severity of neurological dysfunction. GS-2 is often fatal as the patients develop accelerated an hemophagocytic syndrome phase secondary to immunological impairment. Therefore, early recognition of patients with GS-2 and prompt intervention with bone marrow transplant is critical. GS-3 does not require treatment. Subtype 2 is the most common and only 13 cases are reported in the literature from India (1-3), which we have summarized in Table 1 and compared with our case in an attempt to highlight the most consistent findings in GS.

\section{Case Report}

A 20-month-old boy, third in birth order, born of a non consanguineous marriage, with uneventful antenatal and perinatal history, presented to us with complaints 
Table 1. Frequency of clinical and laboratory characteristics of Griscelli syndrome in Indian literature and comparison with our case

\begin{tabular}{|c|c|c|}
\hline Patient characteristics & Frequency $(\mathrm{X} / \mathrm{Y})$ & Present Case \\
\hline Age $(<1$ year $)$ & $4 / 13$ & - \\
\hline Age ( $>1$ year $)$ & $9 / 13$ & 20 months \\
\hline Male & $9 / 13$ & Male \\
\hline Female & $4 / 13$ & - \\
\hline Presence of consanguinity & $4 / 10$ & Absent \\
\hline Fever & $10 / 12$ & Present \\
\hline Abdominal distension & $6 / 10$ & Present \\
\hline Anasarca & $4 / 8$ & Present \\
\hline Jaundice & $5 / 9$ & Present \\
\hline Seizure & $1 / 8$ & Absent \\
\hline Excessively fair skin & $4 / 9$ & Present \\
\hline Developmental delay & $3 / 12$ & Absent \\
\hline Pallor & $10 / 12$ & Present \\
\hline Silvery gray hair, eyelashes, eyebrows & $13 / 13$ & Present \\
\hline Splenohepatomegaly & $9 / 11$ & Present \\
\hline Pancytopenia & $7 / 11$ & Present \\
\hline Bicytopenia (anemia + thrombocytopenia) & $4 / 11$ & Present \\
\hline Intracytoplasmic granules in leucocytes & $0 / 12$ & Absent \\
\hline Hemophagocytosis & $3 / 9$ & Absent \\
\hline$\uparrow$ Triglycerides/ $\downarrow$ Fibrinogen & $5 / 7$ & Present \\
\hline$\uparrow$ Alkaline Phosphatase $/ \downarrow$ albumin & $5 / 10$ & Present \\
\hline Hair shaft microscopy (clumped melanosomes) & $12 / 12$ & Present \\
\hline Skin biopsy & $10 / 10$ & NA \\
\hline Outcome (alive) & $8 / 12$ & Alive \\
\hline
\end{tabular}

$\mathrm{X}$ : Number of cases reported with the characteristic; Y: Number of cases looked for that particular characteristic.

of fever for two months and yellowish discoloration of eyes, body and urine with increasing pallor and weight loss for one month. On Physical examination, his anthropometry parameters were as follows: weight $8.5 \mathrm{~kg}$ (below $3 \mathrm{rd}$ percentile), length $75 \mathrm{~cm}$ (below 3 rd percentile), HC $44 \mathrm{~cm}$ (below 3 rd percentile). On physical examination, child had pallor, icterus, silvery gray hair, white eyelashes, sparse eyebrows and hypopigmented skin (Figure 1).

On abdominal examination, hepatosplenomegaly was detected. The liver was firm; $5 \mathrm{~cm}$ below costal margin with span of $11 \mathrm{~cm}$. Spleen was also firm in consistency and $6 \mathrm{~cm}$ enlarged in splenic axis. In laboratory investigation, complete blood counts (CBC) revealed pancytopenia with hemoglobin $2.7 \mathrm{mg} / \mathrm{dL}$, total leukocyte count $2,200 / \mathrm{mm}^{3}$, absolute neutrophil count $1,190 / \mathrm{mm}^{3}$ and platelet count $18,000 / \mathrm{mm}^{3}$. On peripheral blood examination there was also pancytopenia with no inclusions in granulocytes which was emphasized to rule out chediak higashi syndrome. Liver function tests revealed a serum bilirubin level of $18.16 \mathrm{mg} / \mathrm{dL}$ with direct component of $12.81 \mathrm{mg} / \mathrm{dL}$, Serum albumin 2.0 g/dL, hypertransaminemia (SGOT/SGPT: 236/138 IU/ L), prothombin time $28 \mathrm{sec}$ and INR 1.76. S. Lactate dehydrogenase, S. Triglycerides, S. ferritin, S. fibrinogen values were $1,499 \mathrm{U} / \mathrm{L}, 311 \mathrm{mg} / \mathrm{dL}, 1,053 \mathrm{ng} / \mathrm{mL}, 108$ $\mathrm{mg} / \mathrm{dL}$ respectively. Viral markers for hepatitis were negative.

USG abdomen showed fatty hepatomegaly, splenomegaly and mild ascites. Bone marrow aspiration and biopsy was suggestive of normoblastic marrow,

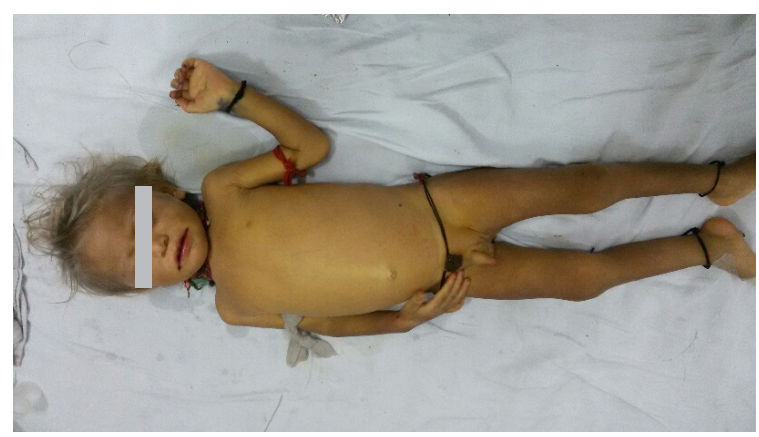

Figure 1. Showing characteristic silvery gray hair, white eyelashes, sparse eyebrows and hypo pigmented skin.

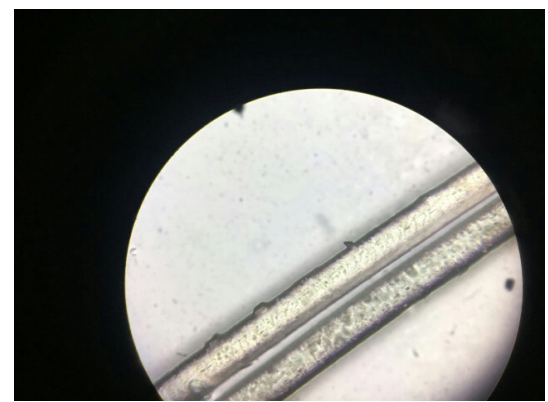

Figure 2. Hair microscopy showing melanosomes clusters present along hair shaft.

and hair microscopy showed presence of melanosome clusters along the hair shaft (Figure 2).

In view of these findings, a diagnosis of GS type 2 was made. For HLH, 5 criteria out of 8 were present (fever, splenomegaly, pancytopenia, raised ferritin and 
hypertriglyceridemia/hypofibrinogenemia) but bone marrow aspiration cytology did not reveal findings of HLH.

Supportive therapy including blood transfusion was given to the child and immunosuppresion therapy (mycophenolate mofetil) was started while bone marrow transplantation was advised. Child is currently under follow up and clinical as well as hematological parameters have improved.

\section{Discussion}

GS was first described by Griscelli and Siccardi in 1978 (4). GS usually manifests in persons aged 4 months to 4 years, though the youngest reported is 1 month with no sex predilection (5).

GS is inherited in an autosomal recessive manner and caused by mutations in one of the three genes. Clinical features of GS depend upon subtype. Hypomelanosis and silvery gray hair are hallmarks of all three GS subtypes. Hypopigmentation of the skin and the hair is accompanied by the presence of large aggregates of pigment in hair shafts and the accumulation of mature melanosomes in melanocytes. GS1 caused by mutations in Myosin-Va gene (MYO5A) presents with neurological involvement without immune dysfunction. GS2 is caused by mutations in the RAB27A gene and is associated with immunological dysfunction without primary neurological impairment. The cytotoxic defect caused by RAB27A mutations is responsible for triggering the hemophagocytic syndrome characterized by acute onset of uncontrolled T-lymphocyte and macrophage activation. Neurological symptoms in GS-2 may be due to infiltration of brain by the activated hematopoietic cells. RAB27A and MYO5A are located at band $15 \mathrm{q} 21(6,7)$. The third form of GS, being the least common of all, is caused by mutation in the gene $M L P H$ that encodes melanophilin and phenotype of this form is limited to characteristic hypopigmentation without neurologic or immunologic abnormalities. In our case, genetic analysis could not be performed owing to the poor affordability of the parents.

The constellation of symptoms of silvery grey hairs and hepatosplenomegaly raised the possibility of Chediak Higashi and GS as primary diagnosis for this child. Absence of inclusions in granulocytes on peripheral blood examination and hair microscopy findings confirmed the diagnosis of GS and ruled out Chediak Higashi syndrome (8). Consistent with symptoms described in subtype 2 , this child also shared features like fever, pallor, jaundice, hepatosplenomegaly, and fulfilled five criteria of HLH - fever, pancytopenia, splenomegaly, raised ferritin $(1,053 \mathrm{ng} / \mathrm{mL})$ and hypertriglyceridemia (triglycerides- $311 \mathrm{mg} / \mathrm{dL}$ ) / hypofibrinogenemia (108 $\mathrm{mg} / \mathrm{dL}$ ). This child did not have any neurological manifestations like seizures, spasticity, or developmental delay, which are usually described in subtype 1 . Hence, the diagnosis of GS-2 with HLH was made.

GS-2, the type reported here, is the most common of all three subtypes. There are only 13 case reports from India of GS-2(1-3), one report of GS-3 (9) with report of no cases of GS-1. Table 1 shows the frequency of clinical and laboratory characteristics of these Indian cases and comparison with our case. The features that were consistently present in all the 13 cases reported, were silvery gray hair, eyelashes, eyebrows; clusters of melanosomes on hair microscopy and hypomelanosis with irregular melanin pigmentation in basal melanocytes on light microscopy of skin. Other frequently occurring features were fever, pallor, hepatosplenomegaly and pancytopenia. Our patient manifested with all the clinical features of GS-2 with HLH although skin biopsy could not be done. Only two previously reported cases $(2,10)$ fulfilled 5 out of 8 criteria to be labeled as HLH, along with documenting hemophagocytosis in bone marrow. In one other case (3), lymphohistiocytosis was demonstrated in an enlarged cervical lymph node, although this case did not meet the criteria of HLH as demonstration of hemophagocytosis alone is neither pathognomonic nor diagnostic for HLH.

Stem cell transplant is the only curative treatment for HLH associated with GS subtype 2 as these children succumb to recurrent infections and secondary neurological involvement (11). Secondary CNS involvement is caused by the infiltration of lymphocytes and histiocytes as a result of hemophagocytic syndrome.

In conclusion, GS is a rare entity, which shares a close clinical spectrum with Chediak-Higashi syndrome and other immunodeficiencies. Often the greatest barrier to a successful outcome is a delay in diagnosis, which is difficult because of the rarity of this syndrome. High index of suspicion about this condition aids in early recognition and foresees the life threatening complications like HLH. Prompt initiation of treatment with bone marrow transplant is essential for the survival of such affected patients.

\section{Acknowledgements}

We are grateful to the parents of the patient who provided their informed consent for images and clinical information to be reported in the journal.

\section{References}

1. Singh A, Garg A, Kapoor S, Khurana N, Entesarian M, Tesi B. An Indian boy with griscelli syndrome type 2: Case report and review of literature. Indian J Dermatol. 2014; 59:394-397.

2. Mahalingashetti PB, Krishnappa MH, Kalyan PS, Subramanian RA, Padhy S. Griscelli sndrome: Hemophagocytic lymphohistiocytosis with silvery hair. J Lab Physicians. 2012; 4:129-130.

3. Rajyalakshmi R, Chakrapani RN. Griscelli syndrome type 2: A rare and fatal syndrome in a South Indian boy. Indian 
J Pathol Microbiol. 2016; 59:113-116.

4. Griscelli C, Durandy A, Guy-Grand D, Daguillard F, Herzog C, Prunieras M. A syndrome associating partial albinism and immunodeficiency. Am J Med. 1978; 65: 691-702.

5. Dotta L, Parolini S, Prandini A, Tabellini G, Antolini M, Kingsmore SF, Badolato R. Clinical, laboratory and molecular signs of immunodeficiency in patients with partial oculo-cutaneous albinism. Orphanet J Rare Dis. 2013; 8:168

6. Ménasché G, Pastural E, Feldmann J, Certain S, Ersoy F, Dupuis S, Wulffraat N, Bianchi D, Fischer A, Le Deist F, de Saint Basile G. Mutations in RAB27A cause Griscelli syndrome associated with haemophagocytic syndrome. Nat Genet. 2000; 25:173-176.

7. Pastural E, Ersoy F, Yalman N, Wulffraat N, Grillo E, Ozkinay F, Tezcan I, Gediköglu G, Philippe N, Fischer A, de Saint Basile G. Two genes are responsible for Griscelli syndrome at the same 15q21 locus. Genomics. 2000; 63:299-306.

8. Valente NY, Machado MC, Boggio P, Alves AC, Bergonse
FN, Casella E, Vasconcelos DM, Grumach AS, de Oliveira ZN. Polarized light microscopy of hair shafts aids in the differential diagnosis of Chédiak-Higashi and GriscelliPrunieras syndromes. Clinics (Sao Paulo). 2006; 61:327332.

9. Khemka P, Kundu R, Niyogi P, Tudu J. A case of silvery hair syndrome: Griscelli syndrome. Indian Journal of Paediatric Dermatology. 2015; 16:72-74.

10. Malhotra AK, Bhaskar G, Nanda M, Kabra M, Singh MK, Ramam M. Griscelli syndrome. J Am Acad Dermatol. 2006; 55:337-340.

11. Cesaro S, Locatelli F, Lanino E, Porta F, Di Maio L, Messina C, Prete A, Ripaldi M, Maximova N, Giorgiani G, Rondelli R, Aricò M, Fagioli F. Hematopoietic stem cell transplantation for hemophagocytic lymphohistiocytosis: A retrospective analysis of data from the Italian Association of Pediatric Hematology Oncology (AIEOP). Haematologica. 2008; 93:1694-1701.

(Received October 22, 2016; Revised December 20, 2016; Accepted December 24, 2016) 